\title{
Neurofeedback as an Intervention to Improve Reading Achievement in Students with Attention- Deficit/Hyperactivity Disorder, Inattentive Subtype
}

\author{
Jeffry P. La Marca ${ }^{1 *}$ and Rollanda E. O'Connor ${ }^{2}$ \\ ${ }^{1}$ Seton Hall University, South Orange, New Jersey, USA \\ ${ }^{2}$ University of California, Riverside, California, USA
}

\begin{abstract}
Research consistently demonstrates that attention deficits have a deleterious effect on academic achievement. Impairments in attention, and not hyperactivity/impulsivity, are associated with learning difficulties and academic problems in students with attention-deficit/hyperactivity disorder (ADHD). To date, most studies have focused on symptoms of hyperactivity/impulsivity, with little research being conducted on interventions for students with ADHD, inattentive subtype. This study examines the use of neurofeedback as an intervention to improve reading achievement in a public school setting. A multiple-baseline-across-participants single-case model was used to assess five fourth-grade students who received 40 daily sessions of neurofeedback. Following the intervention, improvements were observed on objective measures of attention: a continuous performance test (Integrated Visual and Auditory Continuous Performance Test [IVA+Plus]) and/or a test of shifting attention (CNS Vital Signs, Shifting Attention Test [CNS-VS, SAT]). Results on tests of reading fluency revealed little change, although participants demonstrated gains on a measure of reading comprehension (Gray Oral Reading Tests-Fifth Edition [GORT-5]). Results suggest that neurofeedback helped participants to become more accurately engaged with the text with more focused attention to content. Thus, neurofeedback may be a viable option to assist children with attention deficits for improving both attention and reading achievement.
\end{abstract}

Keywords: academic achievement; attention deficit; interventions; neurofeedback; public schools; reading comprehension

Citation: La Marca, J. P., \& O'Connor, R. E. (2016). Neurofeedback as an Intervention to Improve Reading Achievement in Students with Attention-Deficit/Hyperactivity Disorder, Inattentive Subtype. NeuroRegulation, 3(2), 55-77. http://dx.doi.org/10.15540/nr.3.2.55

*Address correspondence to: Jeffry P. La Marca, PhD, Seton Hall University, College of Education and Human Services, 400 South Orange Avenue, South Orange, NJ 07079, USA. Email: Jeff.LaMarca@shu.edu

Copyright: (c) 2016. La Marca and O'Connor. This is an Open Access article distributed under the terms of the Creative Commons Attribution License (CC-BY).
Edited by:

Nancy L. Wigton, PhD, Grand Canyon University, Phoenix, Arizona, USA

Reviewed by:

Scott L. Decker, University of South Carolina, Columbia, South Carolina, USA

Nancy L. Wigton, PhD, Grand Canyon University, Phoenix, Arizona, USA

\section{Introduction}

Attention-Deficit/Hyperactivity Disorder (ADHD) is considered to be among the most widely studied and treated of all psychiatric disorders (American Academy of Pediatrics, 2011; Goldman, Genel, Bezman, \& Slanetz, 1998; Hart, Lahey, Loeber, Applegate, \& Frick, 1995; Volkow et al., 2011). It is a heterogeneous condition characterized by the presence of a variety of symptoms, the most salient of which includes problems with inattention, executive function, impulsivity, memory, and hyperactivity (American Psychiatric Association,
1994). The fourth edition of the Diagnostic and Statistical Manual of Mental Disorders (DSM-IV-TR) recognizes a single disorder that consists of three subtypes: the predominately hyperactive-impulsive subtype, the predominantly inattentive subtype, and the combined subtype where individuals meet criteria for both hyperactivity/impulsivity and inattention (American Psychiatric Association, 2000).

The National Center for Health Statistics reported that $9.0 \%$ of children $(12.3 \%$ of boys and $5.5 \%$ of girls) between the ages of 5 and 17 have been diagnosed with ADHD (Akinbami, Liu, Pastor, \& 
Reuben, 2011). Froehlich et al. (2007) examined prevalence by subtype and found that the majority of students with ADHD meet criteria for the inattentive subtype $(51 \%)$, followed by the combined subtype $(26 \%)$, and then the hyperactive/impulsive subtype $(23 \%)$.

The combined subtype predominates in the literature as the focus of study (Dige, Maahr, \& BackenrothOhsako, 2008; Nigg, 2005), with sparse research focusing solely on the hyperactive/impulsive subtype in isolation from symptoms of inattention. Likewise, the inattentive subtype (i.e., attentiondeficit/hyperactivity disorder without hyperactivity) received little attention until the early 1990s when it was recognized by the American Psychiatric Association (1994).

Although the construct of ADHD has been developed through a medical model, the impact that attention deficits have on students' learning has been studied since the first clinical observations on the topic (American Academy of Pediatrics, 2011; Crichton, 1798; Still, 1902a, 1902b, 1902c). Despite reliance on this medical model to classify attention deficits, addressing the needs of students with ADHD is especially critical in schools, because school is where most children are first identified and their impairments become evident (U.S. Department of Education, Office of Special Education and Rehabilitative Services, \& Office of Special Education Programs, 2008). Research consistently demonstrates that attention deficits have a deleterious effect on academic attainment (Barkley, 2002). Although medical and psychological interventions may be useful, the responsibility for accommodating students with special needs in school ultimately falls to educators.

\section{Attention and Reading Achievement}

Research has indicated that children with ADHD, inattentive subtype, have considerably slower rates of processing speed than both typically developing peers and students with other subtypes (Chhabildas, Pennington, \& Willcutt, 2001; Ghelani, Sidhu, Jain, \& Tannock, 2004). In particular, individuals with the inattentive subtype process visual information slowly and exhibit impairments in allocating attention to information within their visual field (Barkley, Grodzinsky, \& DuPaul, 1992; Swanson, Posner, Potkin, \& Bonforte, 1991). Reading and math disorders, along with other learning disabilities, appear to be more prevalent in individuals with the inattentive subtype than with the predominately hyperactive-impulsive type (Barkley et al., 1992;
Bauermeister, Alegría, Bird, Rubio-Stipec, \& Canino, 1992; Willcutt \& Pennington, 2000).

Ghelani et al. (2004) noted that reading disabilities (RD) and ADHD frequently overlap, and yet few studies specifically address ADHD and reading comprehension. They examined reading rate and comprehension of 96 adolescents, ages 14 to 17 across four groups: students with ADHD only $(n=$ $32)$, RD only $(n=20), \mathrm{ADHD}$ and RD (ADHD/RD; $n$ $=19)$, or a control group $(n=25)$ of typical readers. Study participants were then administered a variety of reading tests. Analysis revealed that all ADHD and/or RD groups scored lower on silent reading comprehension than the control group, and both the $R D$ and $A D H D / R D$ groups scored significantly lower than controls on tests of reading rate and accuracy. The performance of the comorbid ADHD/RD group on tests of reading accuracy and rate was similar to that of the RD group. On reading comprehension, students with ADHD/RD did poorly with silent reading but not with oral reading. These results are similar to another study (Schuck, 2008) that also found that students with ADHD faced difficulties when reading silently, but not orally.

Weiler, Bernstein, Bellinger, and Waber (2000) examined processing speed in children with ADHD, inattentive subtype. Participants included 82 children between the ages of 7 and 11 who met criteria for the inattentive subtype and/or were identified as RD: children with either the hyperactiveimpulsive or combined subtypes were excluded. Additional children were excluded during the screening process if their full-scale IQ was less than 80 , they were taking stimulant medications, or presented with behavioral or emotional problems. Study participants were then subdivided into four groups: ADHD inattentive subtype without RD (ADHD/non-RD), ADHD inattentive subtype with $R D$ (ADHD/RD), RD only, or a fourth group that did not have ADHD or RD. Participants were then administered a battery of timed tests. Findings revealed that while all participants performed less than expected on tasks that measured processing speed, children with ADHD, inattentive subtypes, were significantly slower than the groups without ADHD. Statistically significant differences were found between the ADHD/RD and non-ADHD/RD group when compared on tasks of processing speed, written language, and a test of motor speed: the ADHD/RD group did worse on these tasks.

Neurofeedback and reading achievement. The literature has long noted that neurofeedback produces positive outcomes on a variety of cognitive 
and academic measures (Leins et al., 2007; Linden, Habib, \& Radojevic, 1996; Vernon et al., 2003); however, most neurofeedback studies have been conducted in clinical settings, while few studies have been conducted in $\mathrm{K}-12$ schools. We wished to examine the effects of neurofeedback, particularly improvements in attention, in a public school setting. Moreover, for improvement in attention to matter, it should affect domains known to be important to school achievement. Using the findings of studies that demonstrated deficits in processing speed for visual stimuli (Barkley et al., 1992; Swanson et al., 1991) and reading comprehension (Ghelani et al., 2004) for students with ADHD, we selected reading skills as our academic outcome for neurofeedback training. Moreover, reading serves as the foundation on which many other content areas are built; thus improvements in both attention and reading ability, if found, would establish social validity for the process. Therefore, the intent of our research is to explore the feasibility of an intervention in an elementary school setting, as well as examine if improvement in attention, resulting from neurofeedback translates into gains in academic achievement, thus addressing the most salient concern of educators to maximize opportunities for learning.

At present, only a handful of neurofeedback studies have been conducted in public schools. Wadhwani, Radvanski, and Carmody (1998) published a case study of a middle school student with ADHD and noted improvements on a standardized achievement test. The same research team (Carmody, Radvanski, Wadhwani, Sabo, \& Vergara, 2001) conducted a study in an elementary school of students who exhibited behavioral problems and ADHD; however, their results were inconclusive. Orlando and Rivera (2004) examined the use of neurofeedback to improve reading performance. Their sample included 34 public school students with ADHD in grades six, seven, and eight, with three additional students from a different school in grades one, four, and five. The authors acknowledged the disparity in age of their participants and potential issues concerning the heterogeneity of their sample. Some participants purportedly had "identified learning problems," but these impairments were not identified. Attrition rates were high and standardized procedures were not established for pre- and postassessments. Of the 17 students in the original experimental group, only 12 completed the study. Of those, nine participants' treatment protocols were based on quantitative electroencephalographic (qEEG) evaluations conducted by a volunteer neurofeedback practitioner from the local community; these were used as a "protocol guide" by the primary author. The three remaining students were not assessed with qEEGs and "protocols were developed based upon the clinical decisions made by the psychologist and upon information gained from teachers and/or parents concerning the student's behavior for the week" (Orlando \& Rivera, 2004 , p. 6). Screening procedures also failed to control for comorbid conditions with one participant being jailed just as the study commenced and another reclassified as "mildly mentally retarded." Although the authors concluded that neurofeedback was more effective than no training for improving reading achievement, lack of experimental control draws this finding into question.

Steiner, Frenette, Rene, Brennan, and Perrin (2014) reported a randomized controlled trial in a public school setting that neurofeedback training improved symptoms of inattention and holds promise as an intervention. However, the researchers only used subjective outcome measures including parent and teacher rating scales of attention and behavior and classroom observations. The study did not report on any measures of academic achievement.

Coben, Wright, Decker, and Morgan (2015) reported that coherence-based neurofeedback resulted in "significant gains in reading" following 20 sessions. However, they did not specify what components of the reading process improved (e.g., accuracy, comprehension, fluency, word attack). They noted that all study participants $(n=42)$ "received pre- and post-educational measures focused on reading abilities" that included the administration of the Woodcock-Johnson Tests of Achievement III (WJ III; Woodcock, McGrew, \& Mather, 2007) and the Gray Oral Reading Tests-Fourth Edition (GORT-4; Wiederholt \& Bryant, 2001). However, an issue arises concerning the use of the GORT-4, which has been found to lack both content validity and concurrent validity as a measure of reading comprehension. Keenan and Betjemann (2006) reported that reading comprehension on the GORT4 , using multiple-choice questions that are answered following the reading of graded stories, were not passage-independent. In other words, it is possible to answer many of the questions without having read each passage.

Given the limited research on neurofeedback and academic achievement, we decided to examine its effects in a public school setting. Furthermore, a dearth of research exists in schools even though school is where neurofeedback, arguably, has the potential to have the greatest impact. Our intent 
was to examine what effects, if any, neurofeedback may have on attention and reading achievement.

Our research examined three research questions within the context of examining the efficacy of neurofeedback with students presenting with symptoms of ADHD, inattentive subtype, in a public school setting:

1) Will neurofeedback enhance attention as measured by Continuous Performance Tests (CPTs)?

2) Will neurofeedback improve performance on measures of reading fluency?

3) Will neurofeedback improve performance on measures of reading comprehension?

\section{Method}

\section{Participants}

Participants were selected from students in a relatively affluent suburban coastal community in southern California at an elementary school. In keeping with the requirements of the University of California, Riverside's Human Research Review Board (HRRB), all students had to be referred by school officials. Specifically, the HRRB required that the school psychologist and administrators identify potential candidates (blind to the researcher) for screening based on reviews of educational records. Students with educational histories in which ongoing problems with inattention and distractibility had been noted by current or former teachers and parents, as well as candidates with confirmed medical diagnoses of ADHD, inattentive type, were to be considered. Once a pool of potential candidates was selected, we were able to screen for participants who met inclusionary criteria.

Description of setting. Participants were students in general education classrooms at the Sunny Shoals Elementary School, one of many schools within the large Maritime Unified School District (the name of the school and school district are pseudonymous). The school is located in a relatively affluent suburban coastal community of southern California. During the 2012/2013 school year, 611 students in grades $\mathrm{K}$ to 5 were served by 18 general education classroom teachers and four special education teachers.

As the intent of this study was to examine the use of neurofeedback in a school setting, a space within a special education classroom at Sunny Shoals Elementary School was provided for our research. The classroom was used throughout the day by small groups of students who were pulled out of general education classrooms in order to receive specialized instruction. A dedicated place, segregated from the rest of the classroom, was provided at the back of the room for the latter half of the school year so that all aspects of this study could be conducted during the school day without interruption. The only exception occurred to conduct qEEG assessments. While these were also conducted during the school day, a separate room was used.

Participant selection process. The participant selection process consisted of two phases; an initial phase where a pool of potential participants was identified and administered several instruments designed to determine if they might be appropriate candidates for the study, and a second phase during which participants were exposed to neurofeedback and confirmed final eligibility based on the study's criteria. All assessments throughout the study were conducted at the school site, during regular school hours. The initial target group included 15 students in Grades 3-5, as children of this age have received several years of reading instruction and passed the age-of-onset criterion for ADHD as established by the DSM-IV-TR (APA, 2000). Potential participants who did not meet criteria for ADHD and those with profiles indicative of either the ADHD hyperactive or combined subtypes were excluded.

Once the initial pool of 15 potential candidates had been identified, the school provided each student's parents with an information letter and consent form. The parents of 10 students returned signed consents; one student was in third grade, eight students in fourth, and one in fifth, all between nine and 10 years of age. Following initial screening, three participants (two in fourth grade and one in fifth) did not meet the study's criteria and were excluded. This left seven students to continue with the final phase of screening. A second set of letters and consent forms were sent to the parents of these students, and student participants were asked to sign an assent form. One student's parents declined to give consent, and the third grade student became anxious immediately prior to the beginning of the final assessment (a qEEG evaluation) and withdrew from the study. Of the five students remaining, all completed screening procedures and participated in the study. These five participants included an ethnically diverse group of students consisting of four boys and one girl, all between the ages of nine and 10 (Table 1; the names of all participants are pseudonymous). 


\section{Table 1}

Participant Demographics

\begin{tabular}{ccccccccccc}
\hline Student & Age & Gender & Grade & Ethnicity & $\begin{array}{c}\text { Existing } \\
\text { Diagnosis }\end{array}$ & $\begin{array}{c}\text { Family } \\
\text { History } \\
\text { ADHD }\end{array}$ & $\begin{array}{c}\text { Prescription } \\
\text { Medications }\end{array}$ & $\begin{array}{c}\text { Referred } \\
\text { for } \\
\text { IEP/504 }\end{array}$ & $\begin{array}{c}\text { Eligible } \\
\text { for } \\
\text { Services }\end{array}$ & $\begin{array}{c}\text { Teacher } \\
\text { Referral }\end{array}$ \\
\hline Mildred & 9.58 & $\mathrm{~F}$ & 4 & Hispanic & No & Yes & No & No & No & Yes \\
Dudley & 10.63 & M & 4 & Black & Yes & No & No & 504 & Yes & Yes \\
Nimrod & 9.37 & M & 4 & Vietnamese & No & No & No & No & No & Yes \\
Webster & 10.66 & M & 4 & White & No & Yes & No & No & No & Yes \\
Egbert & 9.98 & M & 4 & Hispanic & Yes & No & No & IEP & No & Yes \\
\hline
\end{tabular}

Note. Age calculated at the time of the study.

Selection criteria. In addition to the age/grade, consents, expressed interest, and school attendance requirements, students selected for the study met the following inclusionary criteria:

1) Ratings by a parent and/or a teacher on an ADHD rating scale that exceeding the cutoff for an attention deficit (T-scores $\geq 61$ on the Conners 3ADHD Index, Parent and Teacher Rating Scales, described below),

2) Demonstrated impaired performance on a CPT that was consistent with ADHD (determined by proprietary algorithms used by the IVA+Plus, described below),

3) A full-scale intelligence quotient $(F S \mid Q) \geq 80$, and

4) Theta/beta ratios $\geq 2.0$ (theta $=4$ to $8 \mathrm{~Hz}$, beta $=15$ to $18 \mathrm{~Hz}$ ) at $\mathrm{Cz}$.

The presence of comorbid conditions (e.g., seizure activity, brain injury, psychiatric conditions such as anxiety, depression, or other brain-based impairments) would have resulted in exclusion from participation; however, no potential candidates were excluded for these reasons.

Neurofeedback software and equipment.

Neurofeedback system. SmartMind Pro (Sandford, 2012), consisting of an EEG software application and a two-channel EEG amplifier, was used to provide neurofeedback to students. The software ran on a laptop computer using Microsoft's Windows 7 operating system that was connected to the amplifier. EEG was measured using gold-plated disk recording electrodes and ear clips.

The neurofeedback software displays each participant's EEG in real time with output customizable to show only the bandwidths selected for training. Neurofeedback is accomplished using specially designed computer games. Although some of the neurofeedback games require the use of a mouse, only those that used EEG were implemented to avoid variability that might be attributed to operating the computer through physical activity. The software records and maintains information about each activity within a session; these data include the mean amplitude of EEG bandwidths being trained in $\mathrm{Hz}$, standard deviation of each frequency band, and session time.

The neurofeedback system was used during the final stage of screening to identify potential participants with elevated theta/beta ratios. Studies have shown that higher ratios are particularly observable over the frontal and central midline brain regions. Previous research has suggested that elevated ratios are an electrophysiological indicator found in EEGs of individuals with ADHD (Monastra et al., 2005; Snyder \& Hall, 2006). Research has reported that the individuals with ADHD who benefit most from neurofeedback are those with elevated theta/beta ratios (Monastra, Monastra, \& George, 2002).

qEEG software and equipment. The $q E E G$ assessments were conducted using WinEEG software developed by Mitsar Co. Ltd. (Saint Petersburg, Russia). Data were collected with a 21channel Mitsar EEG-201 amplifier. Similar to the equipment used with the neurofeedback system, gold-plated disk EEG recording electrodes and ear clips were at all 19 standardized scalp locations established by the International 10/20 System (Jasper, 1958). Following each assessment data were compared with the Human Brain Institute (Saint Petersburg, Russia) normative database. 
Measures

Screening measures. Participant selection was based on criteria that identified students with profiles consistent with the definition of ADHD, as defined by the DSM-IV-TR. Children with an existing diagnosis (made by a qualified medical professional) of ADHD, Inattentive Subtype, were considered for inclusion. As there are no "gold standards" for the identification of children with attention deficits, several measures were used for participant selection.

Student health history. Parents completed a student health history questionnaire, which included medical history and checked for an existing diagnosis of ADHD. Students diagnosed with the inattentive subtype were considered and those diagnosed with either the hyperactive/impulsive or combined subtypes were excluded. Two participants had been previously diagnosed with ADHD, and two additional students had parents indicate a family history of the disorder.

Parents of participants were asked at the onset of the study to disclose if their child was receiving pharmaceutical interventions. Potential participants were excluded from the screening process if they received pharmaceutical or other independent medical interventions for ADHD, especially if they received psychotropic medications (i.e., stimulant or other prescription medications). In the event that participants began medical interventions during the study, parents were asked to disclose this information because modifications, changes, and titrations of medications could affect progress monitoring and the results on outcome measures.

School records. Additional data were gathered on whether each student had been referred by a teacher for possible participation in special education programs, had been recommended for an Individualized Educational Program (IEP), or a plan under Section 504 of the Rehabilitation Action of 1973 (Section 504), and had been found eligible for services. Although all students had received teacher referrals for special education services, only two had been recommended for IEP/Section 504 plans, and just one had been found eligible. All teachers reported that referred students had problems with attention in the classroom environment. None of the participants received specialized reading instruction.

Rating scale. The Conners 3 ADHD Index (Conners 3Al; Conners, 2008a) is a screening instrument designed to differentiate ADHD children, ages 6 to 18, from typically developing peers and requires approximately five minutes to administer. There are separate forms for parents (Conners 3Al$\mathrm{P})$ and teachers (Conners 3Al-T); both contain 10 questions. T-scores $\geq 61$ suggest responses describing children with ADHD (Conners \& Research and Development Department, 2009). Participants were considered for inclusion if scores from either a parent or a teacher exceeded a T-score of 61 . The ranges of internal reliability on the subtests for ages 6 to 9 are from .91 to .94 . Test-retest reliability ranged from .84 to .93 . The inter-rater reliability coefficient between parent and teacher forms is .85 . The sensitivity of the $3 \mathrm{Al}-\mathrm{P}$ is $88 \%$ and the $3 \mathrm{Al}-\mathrm{T}$ is $79 \%$ (Conners, 2008b). For this study, if there was a discrepancy between raters and just one rater (parent or teacher) indicated that a potential participant's score exceeded the cutoff, screening continued with other measures to determine if the student's profile was congruent with a diagnosis of ADHD.

Continuous performance test. The Integrated Visual and Auditory Continuous Performance Test (IVA+Plus; Sandford \& Turner, 2007) is a 13-minute CPT that uses both visual and auditory prompts to provide an objective measure of behaviors that are associated with the core symptoms of ADHD. A study of the IVA+Plus' validity reveals a sensitivity of $92 \%$, specificity of $90 \%$, and a concurrent validity with other diagnostic instruments (Test of Variables of Attention CPT [TOVA], the Conners Abbreviated Symptom Questionnaire, and the Conners Rating Scales) ranging from $90 \%$ to $100 \%$ (Sandford \& Turner, 2009). Test-retest reliability has a range of .66 to .75 for Attention Quotient scores (AQ; inattention) and .37 to .41 for Response Control Quotient scores (RCQ; hyperactivity/impulsivity). Concurrent validity with other CPTs including the TOVA is 0.9. Potential participants with scores that indicated an attention deficit were considered for the study; proprietary algorithms used by the IVA+Plus Interpretive Flowchart that suggested a diagnosis of ADHD were used as one component of the participant selection process. Test results generate a Combined Sustained Attention (C-SA) score derived from Auditory Sustained Attention (A-SA) and Visual Sustained Attention (V-SA).

Intelligence screening. The Wechsler Abbreviated Scale of Intelligence-Second Edition (WASI-II; Wechsler, 2011) is a 15-min intelligence test for individuals ages 6 to 90 that provides estimates of Verbal IQ (VIQ), Performance IQ (PIQ), and FSIQ. For children ages 8 to 9 , split-half reliabilities range from .85 to .91 for the subtests and .90 to .96 for the IQ scores. Concurrent validity with the WISC-IV, 
have correlations ranging from .73 to .83 on the subtests and .79 to .91 for the IQ scores. A FSIQ $\geq$ 80 was used as a criterion for participants to be included in this study. All participants met criteria for FSIQ, with IQ estimates ranging from 90 to 107.

Screening for comorbid reading disabilities. The Woodcock Reading Mastery Test, Third Edition (WRMT-III; Woodcock, 2011) was used as a screening device to assess for the possibility of comorbid reading disabilities and as a measure of reading achievement. Results from the WRMT-III indicated that participants' Total Reading (standard) scores, derived from the Basic Skills and Reading Comprehension cluster scores ranged from 84 to 112. Oral Reading Fluency standard scores ranged from 85 to 100 . One student, Webster, obtained high scores on several of the WRMT-III subtests and had a Reading Comprehension cluster score of 124 . His Oral Reading Fluency score, however, was 96. Although Webster appeared to be a good reader, this study's exclusionary criteria did not address ceilings on screening instruments and, as this participant met criteria on all other measures, he was retained as a participant.

\section{Baseline and outcome measures.}

Measure of reading achievement. The Gray Oral Reading Tests-Fifth Edition (GORT-5; Wiederholt \& Bryant, 2012a) is a standardized norm-referenced test of oral reading skills that provides measures of rate, accuracy, fluency, and comprehension. Students are presented with a series of passages that increase in difficulty. Rate and accuracy are scaled scores (scaled from 1 to 20 with a mean of 10 and a $S D=3$ ) derived from the speed with which each passage is read in seconds and the number of words read correctly, respectively. The fluency score is derived from the rate and accuracy scores. Comprehension is a scaled score derived from correct responses to open-ended passagedependent questions. An Oral Reading Index (ORI) provides a composite score derived from the fluency and comprehension scores.

The GORT-5 has two alternate forms; both forms require approximately 15 to 45 minutes to administer (Wiederholt \& Bryant, 2012b). The reliability coefficients for the subtest scores on each form exceed > .85; the ORI coefficient on each from is .96 and .97 , respectively. Test-retest reliability is .82 to .90 . When one form was administered, followed by the alternate form, the test-retest reliability is .77 to .88 (Hall \& Tannebaum, 2013; Wiederholt \& Bryant, 2012b). In order to minimize potential issues with practice effect, Form 1 was used at pretest,
Form 2 was used at posttest, and Form 1 was again used at follow-up.

qEEG assessment. qEEG assessments provide high temporal resolution of EEG activity and deliver low resolution "maps" of brain function. Chabot and Serfontein (1996) noted that qEEGs have a specificity of $88.8 \%$ and a sensitivity of $93.7 \%$ in distinguishing children with ADHD from typically developing others. Thus, qEEGs have diagnostic utility as part of the process for identifying children with ADHD. As these assessments must be conducted by qualified professionals and require considerable expertise to interpret; only the final set of candidates being considered as participants were evaluated. These qEEGs were also used as a baseline measure. Data obtained from the qEEG assessments were considered when developing the neurofeedback training protocols that addressed the unique EEG profiles of each participant.

qEEG evaluations were the last assessments to be done. For this procedure, electrodes were placed on participants at each of the 19 locations on the scalp with linked-ear reference. Interpretations of qEEG results were evaluated by a third-party expert in qEEGs and a medical doctor (both from Brain Science International, San Ramon, California), and then approved by a clinical psychologist who is an expert in qEEG evaluations and the use of neurofeedback for the treatment of ADHD. Individualized protocols were developed for each participant with the intent to maximize the efficacy of the neurofeedback training. Following completion of the study, qEEGs were again administered to each participant and pre- and post-intervention results were compared.

Progress monitoring measures. Participants had their progress monitored throughout the study on measures of attention, reading comprehension, and reading fluency. Specifically, following completion of each 30-min neurofeedback session, participants were administered three instruments, described below.

CNS Vital Signs (CNS-VS; Gualtieri \& Johnson, 2006) is a battery of computerized neurocognitive tests (CNT) that includes a Shifting Attention Test (SAT), which measures attention during progress monitoring and also provides a measure of executive function that may indicate the presence of an attention deficit (Gualtieri \& Johnson, 2006). Scores are provided for correct responses, number of errors, and correct reaction time in milliseconds. The test-retest reliability of the SAT for ages 7 to 90 
(based on a normative sample, $n=99$ ) with a median interval of 27 days ranges from .69 to .80 (Gualtieri \& Johnson, 2006).

The Dynamic Indicators of Basic Early Literacy Skills (DIBELS; Good \& Kaminski, 2003) test of Oral Reading Fluency (ORF) is a standardized measure of reading rate and accuracy. This task requires students to read aloud for $1 \mathrm{~min}$ from graded passages. Scores are calculated based on the total number of words read per minute minus the number of errors. Alternate form reliability is .92, test-retest reliability is .92 to .97 , and concurrent validity with other tests is .80 (Shanahan, 2005). There are 30 DIBELS ORF reading passages available from the publisher. As the number of probes required for the study exceeded those available, two editions of the ORF were used (each contained a different set of 30 passages) with passages from each alternated every other session. All participants received fourthgrade passages with the exception of Webster, who received the eighth-grade set. Participants were asked to read for $1 \mathrm{~min}$ and their results recorded. Four participants were monitored using fourth-grade passages presented in the same order, while Webster received eighth-grade passages as his reading abilities were above grade level.

The AIMSweb Reading Curriculum-Based Measurement (R-CBM) Maze (Maze; Shinn \& Shinn, $2002 a$ ) is a multiple-choice cloze task intended to serve as a measure of reading comprehension. The Maze requires participants to read silently for $3 \mathrm{~min}$. The first sentence is complete. Every 7th word after that is replaced with a set of three words of which only one is correct. Participants are asked to select the correct word; correct and incorrect responses are counted to obtain raw scores (Shinn \& Shinn, 2002b). Validity coefficients range from 0.60 to 0.80 (Shinn \& Shinn, 2002a). The test-retest reliability for Grades 1-7 has a range of .66 to .91 (National Center on Response to Intervention, 2012). There are 24 Maze passages available from the publisher but the number of probes required during the study exceeded 40; these included the sessions required to establish baseline. To address this issue, the 24 passages were presented in sequence. They were then randomly reordered and repeated. Four participants were presented with the same fourthgrade passages in the same order; Webster received eighth-grade passages.

\section{Procedures}

Research design. Studies using single-case design (SCD) have been of considerable utility in the development of evidence-based practices in special education (Horner et al., 2005; Kennedy, 2005; Kratochwill et al., 2010), applied and clinical psychology (Chambless \& Hollon, 1998; Gustafson, Nassar, \& Waddell, 2011), and within the field of neurofeedback (Kratochwill et al., 2010). By examining whether experimental control of an independent variable produces a consistent effect on a dependent variable, SCDs can determine whether a functional relation exists between the two (Kennedy, 2005). Individual performance of each participant is examined prior to, during, and after the intervention (Horner et al., 2005). Although disagreements exist regarding the minimum number of participants required within a SCD to lend support that an intervention is efficacious, Chambless and Hollon (1998) suggest that three or more are required, along with replication of the study from another independent research site, to suggest that the treatment is "possibly efficacious."

This study used a multiple-baseline-acrossparticipants SCD model. This model requires that participants begin the initial baseline phase at the same time and they are then staggered into the intervention phase, such that each participant not only serves as his or her own control but is also the unit of analysis (Horner et al., 2005). By staggering the introduction of additional participants, researchers are able test whether the effect of the intervention on a single case replicates multiple times and therefore permits within- and betweenparticipant comparisons (Kratochwill et al., 2010). Doing so helps control for threats to internal validity (Horner et al., 2005). Kratochwill et al. (2010) state that staggering participants also permits causal inferences to be made on the effect of the intervention on the outcomes.

Neurofeedback training based on qEEG-guided protocols is the independent variable. Reading achievement (as measured by scores on the GORT5, AIMSweb Maze, and DIBELS ORF) and attention (as measured by the IVA+Plus and SAT) serve as the dependent variables. Pre- and post-intervention qEEG maps were compared to examine changes in brain function. Participants selected during the screening process were randomly assigned to one of three sets (Cohort 1, 2, and 3), with two participants in the first two cohorts and one in the last. 
Baseline phase. All five participants began the baseline phase at the same time. During this phase, EEG assessment commenced and students were introduced to the neurofeedback equipment and software. After ensuring good connections, EEG was monitored for 3 min using an eyes-open condition. Although monitoring continued throughout baseline, participants did not receive neurofeedback training.

Progress monitoring also commenced during this phase and each participant was assessed on a daily basis with the Maze, ORF, and SAT. Once Cohort 1 had established a stable EEG theta/beta ratio, they proceeded to the intervention phase where they received $30 \mathrm{~min}$ of neurofeedback training, five days per week, for 40 sessions. In the event of absences or other unforeseen circumstances, training continued until 40 sessions were completed.

Intervention phase. During the first week of the intervention phase, participants received an additional $4 \mathrm{~min}$ of training each day to reduce electromyographic (EMG) artifact caused by facial muscle movement. As mean amplitudes of EEG bandwidths fluctuate throughout the day, as well as from day-to-day, the neurofeedback system used provides an automated assessment of EEG to calibrate neurofeedback training goals to adjust for these differences. During this study, a 3-min assessment was conducted at the beginning of each session; the software evaluated the current mean amplitudes of bandwidths being trained and adjusted daily goals accordingly.

During neurofeedback training, participants received rewards that were both visual and aural: Visual rewards were provided in the form of an animated figure moving across on the computer monitor driven by the amplitude of the participant's EEG, and aural rewards were provided by the presence of music or other sounds to indicate success. Failure to meet goals resulted in no (or reduced) movement and sound. Meeting goals for both bandwidths (e.g., theta and beta) simultaneously resulted in faster movement of the animation and increased the volume of sound/music. Each neurofeedback game used the neurofeedback system's default setting to allow participants to successfully meet goals for each bandwidth 84 percent of the time, and both bandwidths simultaneously 71 percent of the time. These goals were set each day, prior to the training, based on the 3-min assessment of each participant's EEG.

When visual assessment of the EEG of one or more participants in Cohort 1 indicated change in the desired direction (e.g., an increase in amplitude of SMR/beta and decrease in theta), Cohort 2 began receiving the intervention. This process was repeated until all cohorts had been staggered in.

Neurofeedback protocols. This study was designed to use theta/beta ratio training protocols, with all participants being trained to inhibit theta and enhance SMR/beta, as first described by Lubar (1991). We used the theta/beta protocol in which theta $(4$ to $8 \mathrm{~Hz}$ ) is suppressed and beta (16 to 20 $\mathrm{Hz}$ ) is enhanced (Monastra et al., 2005). The first 10 sessions used standardized theta/beta protocols for all participants, after which qEEG-guided protocols were used for the final 30 sessions of the intervention, which addressed the unique EEG profiles of each participant to maximize the efficacy of the neurofeedback training (Table 2). The qEEGguided protocols were designed to normalize qEEG. 


\section{Table 2}

Neurofeedback Training Protocols Used During the Study

\begin{tabular}{|c|c|c|c|c|c|c|c|c|}
\hline Participant & Phase & $\begin{array}{c}\text { \# of } \\
\text { Sessions }\end{array}$ & Training & Active & Reference & Enhance $(\mathrm{Hz})$ & \multicolumn{2}{|c|}{ Inhibit $(\mathrm{Hz})$} \\
\hline \multirow[t]{3}{*}{ Mildred } & 1 & 10 & Beta & $\mathrm{Cz}$ & $\mathrm{A} 1$ & 15 to 18 & 4 to 8 & 18 to 30 \\
\hline & 2 & 20 & SMR & C4 & T5 & 12 to 15 & 4 to 10 & 18 to 30 \\
\hline & 3 & 10 & Dual Inhibit & $\mathrm{Fz}$ & $\mathrm{Pz}$ & N/A & 4 to 10 & 18 to 30 \\
\hline \multirow[t]{3}{*}{ Dudley } & 1 & 10 & Beta & $\mathrm{Cz}$ & $\mathrm{A} 1$ & 15 to 18 & 4 to 8 & 18 to 30 \\
\hline & 2 & 20 & SMR & T6 & $\mathrm{Cz}$ & 12 to 15 & 4 to 12 & 18 to 30 \\
\hline & 3 & 10 & Dual Inhibit & $\mathrm{Fz}$ & $\mathrm{A} 1$ & $\mathrm{~N} / \mathrm{A}$ & 4 to 12 & 18 to 30 \\
\hline \multirow[t]{3}{*}{ Nimrod } & 1 & 10 & Beta & $\mathrm{Cz}$ & $\mathrm{A} 1$ & 15 to 18 & 4 to 8 & 18 to 30 \\
\hline & 2 & 20 & SMR & $\mathrm{C} 4$ & T5 & 12 to 15 & 4 to 12 & 18 to 30 \\
\hline & 3 & 10 & Beta & $\mathrm{Fz}$ & $\mathrm{A} 1$ & 15 to 18 & 4 to 12 & 18 to 30 \\
\hline \multirow[t]{3}{*}{ Webster } & 1 & 10 & Beta & $\mathrm{Cz}$ & A1 & 15 to 18 & 4 to 8 & 18 to 30 \\
\hline & 2 & 20 & SMR & T6 & $\mathrm{Cz}$ & 12 to 15 & 4 to 12 & 18 to 30 \\
\hline & 3 & 10 & Beta & $\mathrm{Fz}$ & $\mathrm{A} 1$ & 15 to 18 & 4 to 12 & 18 to 30 \\
\hline \multirow[t]{3}{*}{ Egbert } & 1 & 10 & Beta & $\mathrm{Cz}$ & $\mathrm{A} 1$ & 15 to 18 & 4 to 8 & 18 to 30 \\
\hline & 2 & 20 & SMR & $\mathrm{Cz}$ & $\mathrm{A} 1$ & 12 to 15 & 4 to 12 & 18 to 30 \\
\hline & 3 & 10 & Dual Inhibit & $\mathrm{Fz}$ & $\mathrm{A} 1$ & $\mathrm{~N} / \mathrm{A}$ & 4 to 12 & 18 to 30 \\
\hline
\end{tabular}

Note. For Phase 1, all participants received the same protocol; Phases 2 and 3 used customized qEEG-guided protocols. SMR = Sensorimotor Rhythm; Dual Inhibit = training protocol where two bandwidths are inhibited and no bandwidth is enhanced.

During the establishment of baseline, EEG recordings were made with a monopolar montage using an active electrode placed at $\mathrm{Cz}$ (top center on the scalp) as this location is considered optimal for training (Lubar, 1991). Reference and ground electrodes were placed at $\mathrm{A} 1$ and $\mathrm{A} 2$, respectively. Mean amplitudes of each participant's theta (4 to 8 $\mathrm{Hz}$ ) were recorded using an eyes-open condition for $3 \mathrm{~min}$ per session. Two subsets of the beta bandwidth (15 to $18 \mathrm{~Hz}$ and 16 to $20 \mathrm{~Hz}$ ) were also monitored as both of these have been reported in the literature (Gruzelier \& Egner, 2005; Monastra et al., 2005). Following the completion of three baseline sessions with all participants, theta/beta ratios were calculated using each of the two beta bandwidths recorded and compared. It was found that for all participants, theta/beta ratios were higher when calculated with the beta bandwidth at 15 to 18 $\mathrm{Hz}$. Given that reductions in the theta/beta ratio are associated with increased attentiveness, the decision was made to provide all participants with 10 sessions of neurofeedback in which theta $(4$ to $8 \mathrm{~Hz})$ was inhibited and beta (15 to 18) was enhanced.

Students in all cohorts received the same protocol for the first phase, while the second and third phases were customized based on individual qEEG profiles. Neurofeedback sessions were provided each school day at approximately the same time until every participant had received 40 sessions. Absences, field trips, and special events were accounted for, and students who missed sessions continued with the intervention until they had completed 40 sessions.

Incentives. Neurofeedback can be engaging, especially for motivated adults and adolescents who find that training is intrinsically rewarding and perceive it as a positive way to reduce symptoms and achieve control over unwanted behaviors (Rossiter, 2002). Others, particularly children who do not yet understand the implications of the disorder or the potential for long-term benefits associated with neurofeedback to alleviate symptoms associated with ADHD, can find that their interest in training wanes after the novelty of the invention dissipates and becomes routine. Thus, a reward system was established that was noncontingent on performance but as an incentive to complete each daily session. Initially, students were provided with a chart and for each day that they responded in the affirmative to the question, "Did you try your best today?" were permitted to select a shiny metallic star sticker to record their 
participation. At the end of each week, students who received stars each day earned a "Friday Surprise"-a small reward valued at $\leq \$ 1$.

\section{Follow-up}

Given the important role that reading achievement plays in academic success, as well as the amount of time required for students to receive 40 sessions of neurofeedback during the school day, there is a need to examine the robustness of the intervention over time. In order to do so, each participant was reevaluated after summer vacation and near the beginning of the following school year. Specifically, the Conners 3Al, the GORT-5, and the IVA+Plus were administered. All follow-up results were calculated based on norms that reflected the age of each of the participants, as well as their advancement to fifth grade. As noted previously, Form 1 of the GORT-5 was used.

\section{Data Analysis}

SCD traditionally relies on systematic visual analysis of data, in which relations between the independent and dependent variables are sought, as well as the strength of the relation between them (Horner et al., 2005; Kennedy, 2005; Kratochwill et al., 2010). As data are gathered, they are plotted and visually inspected to determine if a causal relation can be inferred by changes in the outcome that is attributable to manipulations of an intervention. Effects can be demonstrated when there are observable changes between consecutive phases (i.e., baseline and intervention) that differ from what is expected due to manipulation of the independent variable.

\section{Results}

Between the onset of the baseline phase and completion of the intervention phase, participants received 43 to 49 daily sessions; variation in the number of sessions was due to differential baseline phase lengths. The intervention was divided into three phases with all students receiving the same theta beta reduction protocol during Phase 1: inhibit theta (4 to $8 \mathrm{~Hz}$ ) and enhance beta (15 to $18 \mathrm{~Hz}$ ) for the first 10 sessions. Phases 2 and 3 used qEEGguided protocols and contained 20 sessions and 10 sessions, respectively. Progress was monitored using Maze, ORF, and SAT. Post-intervention and follow-up outcomes were measured using the Conners 3AI, the IVA+Plus, and the GORT-5.

\section{Attention Measures}

CNS-VS SAT results. Visual examination of the results for the CNS-VS SAT across all phases revealed that three participants increased in correct responses with neurofeedback and two participants (Mildred and Nimrod) neither increased nor decreased their performance (Figure 1). All participants reduced their errors over the same period. Performance pertaining to reaction time was mixed; three participants, Dudley, Webster, and Egbert demonstrated improved (faster) performance, while Mildred and Nimrod performed slower over time. All students increased their accuracy; the mean percentage of correct responses at pretest was $70.22 \%$; that increased to $87.06 \%$ during Phase 3.

Conners $3 \mathrm{Al}$ results. Both parent and teacher ratings on the Conners $3 \mathrm{Al}$ showed improvements for most participants on all measures (Table 3). The one exception was Nimrod, whose parent gave him a raw score of zero at pretest and posttest. Nimrod's teacher, however, indicated a large improvement with his raw score dropping from 18 on the pretest, to 0 on the posttest. The mean raw score for all participants on the parent scale at pretest was $11.20(S D=6.72)$, which dropped to $6.20(S D=4.55)$ at posttest. Similar declines in scores were noted on the teacher ratings; the mean raw pretest score was $15.60(S D=2.88)$ which dropped to $8.4(S D=5.86)$ at posttest. 


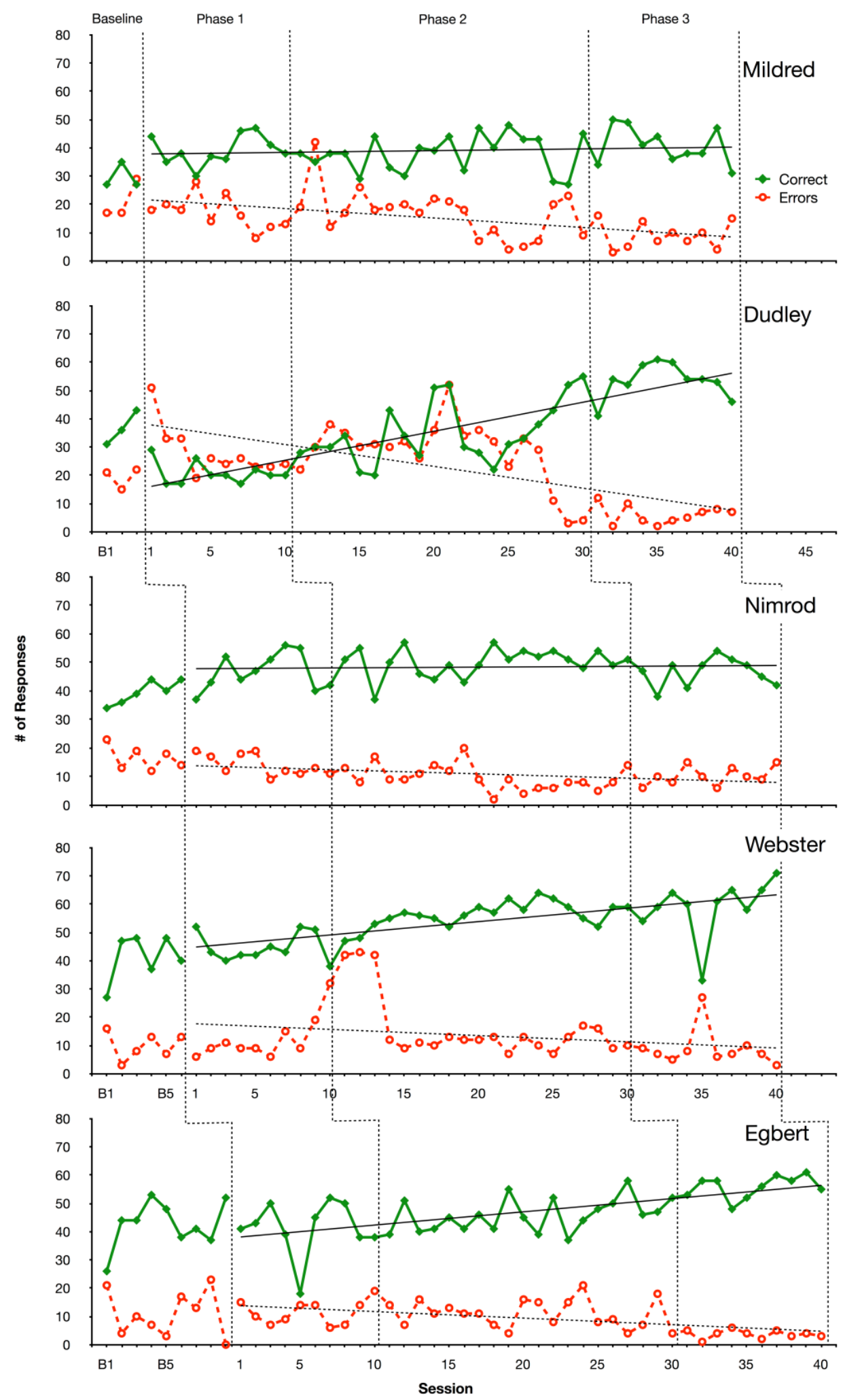

Figure 1. CNS-VS SAT correct responses and errors, trends across all phases. Trends that were expected to increase are represented by a solid line; trends that were expected to decrease are represented by a dotted line. The baseline phase commenced on the same day with participants receiving no more than one session per day. 


\section{Table 3}

Conners 3AI Pretest, Posttest, and Follow-up Scores

\begin{tabular}{|c|c|c|c|c|c|c|c|c|}
\hline & & \multicolumn{5}{|c|}{ Participant } & \multicolumn{2}{|c|}{ All Participants } \\
\hline \multicolumn{9}{|l|}{ Conners 3Al-Parent } \\
\hline \multirow{3}{*}{ Raw Score } & Pretest & 16 & 10 & 0 & 16 & 14 & 11.20 & 6.72 \\
\hline & Posttest & 8 & 9 & 0 & 3 & 11 & 6.20 & 4.55 \\
\hline & Follow-up ${ }^{b}$ & 19 & 10 & 0 & 1 & 8 & 6.20 & 8.23 \\
\hline \multirow[t]{3}{*}{ Probability (\%) } & Pretest & 99 & 91 & 11 & 99 & 99 & & \\
\hline & Posttest & 82 & 87 & 11 & 51 & 94 & & \\
\hline & Follow-up ${ }^{b}$ & 99 & 91 & 11 & 29 & 82 & & \\
\hline T-score & Pretest & $\geq 90$ & $\geq 90$ & 45 & $\geq 90$ & $\geq 90$ & & \\
\hline \multicolumn{9}{|l|}{ Conners 3Al-Teacher } \\
\hline \multirow[t]{3}{*}{ Raw Score } & Pretest & 18 & 12 & 18 & 13 & 17 & 15.60 & 2.88 \\
\hline & Posttest & 13 & 10 & 0 & 5 & 14 & 8.40 & 5.86 \\
\hline & Follow-up ${ }^{b, c}$ & 7 & 4 & 0 & 0 & 3 & 2.80 & 2.95 \\
\hline \multirow[t]{3}{*}{ Probability (\%) } & Pretest & 97 & 89 & 97 & 91 & 96 & & \\
\hline & Posttest & 91 & 84 & 19 & 64 & 92 & & \\
\hline & Follow-up ${ }^{b, c}$ & 73 & 58 & 19 & 19 & 52 & & \\
\hline T-score & Pretest & $\geq 90$ & $\geq 90$ & $\geq 90$ & $\geq 90$ & $\geq 90$ & & \\
\hline \multirow[t]{2}{*}{$(\text { Cutoff } \geq 61)^{a}$} & Posttest & $\geq 90$ & 86 & 45 & 65 & $\geq 90$ & & \\
\hline & Follow-up ${ }^{b, c}$ & $\geq 90$ & 62 & 44 & 44 & 57 & & \\
\hline
\end{tabular}

Note. Posttest and follow-up results in bold indicate change in the desired direction over previous score. Probability (\%) $=$ percentage of time that children in the norming sample with the same score had a diagnosis of ADHD as opposed to typically developing children (Conners \& Research and Development Department, 2009).

${ }^{a}$ The maximum T-Score reported by the test developers is $\geq 90$. ${ }^{b}$ Follow-up was conducted approximately five and a half months after posttest. ${ }^{\circ}$ Conners $3 \mathrm{Al}-$-Teacher follow-up ratings were completed by fifth-grade teachers (pretest and posttest ratings were completed by fourth-grade teachers).

IVA+Plus results. At posttest, all participants demonstrated improvements in their Combined Sustained Attention scores, except for Dudley (Table 4). While this participant appeared engaged during the test, he was observed responding very quickly to the target. His scores were inconsistent from those obtained at pretest. An examination of these posttest results revealed tremendous variability with standard scores ranging from 0 to 157 . These scores suggest that this participant was not motivated to do his best and therefore his IVA+Plus scores for the posttest administration must be viewed with caution. Even when Dudley's scores are considered, group results are positive. For all participants, the group mean scores on the C-SA scale at pretest was $58.60(S D=27.47)$, which increased to $68.40(S D=35.59)$ at posttest; the ASA scale at pretest was $61.00(S D=35.85)$ and increased to $67.80(S D=35.95)$ at posttest; and the V-SA scale was $64.00(S D=29.81)$ at pretest and increased to $74.80(S D=29.06)$ at posttest. At posttest, the algorithms used by the IVA+Plus Interpretive Flowchart no longer suggested a diagnosis for ADHD for two students, Nimrod and Webster, while a diagnosis continued to be suggested for Mildred, Egbert, and Dudley. 


\section{Table 4}

IVA+Plus Pretest and Posttest Sustained Attention Standard Scores

\begin{tabular}{|c|c|c|c|c|c|c|c|c|}
\hline & & \multicolumn{5}{|c|}{ Participant } & \multicolumn{2}{|c|}{ All Participants } \\
\hline & & Mildred & Dudley $^{a}$ & Nimrod & Webster & Egbert & Mean & $S D$ \\
\hline \multicolumn{9}{|l|}{ Subtest } \\
\hline \multirow[t]{3}{*}{ C-SA } & Pretest & 42 & 28 & 91 & 84 & 48 & 58.60 & 27.47 \\
\hline & Posttest & 70 & 7 & 96 & 87 & 82 & 68.40 & 35.59 \\
\hline & Follow-up ${ }^{b}$ & 73 & 47 & 94 & 107 & 66 & 77.40 & 23.49 \\
\hline \multirow[t]{3}{*}{ A-SA } & Pretest & 10 & 52 & 83 & 105 & 55 & 61.00 & 35.84 \\
\hline & Posttest & 55 & 10 & 92 & 92 & 90 & 67.80 & 35.95 \\
\hline & Follow-up ${ }^{b}$ & 83 & 45 & 88 & 110 & 69 & 79.00 & 24.05 \\
\hline \multirow[t]{3}{*}{ V-SA } & Pretest & 80 & 21 & 100 & 67 & 52 & 64.00 & 29.81 \\
\hline & Posttest & 88 & 25 & 100 & 84 & 77 & 74.80 & 29.06 \\
\hline & Follow-up ${ }^{b}$ & 73 & 61 & 101 & 103 & 71 & 81.80 & 19.01 \\
\hline \multirow[t]{3}{*}{ Supports Diagnosis $^{c}$} & Pretest & Yes & Yes & Yes & Yes & Yes & & \\
\hline & Posttest & Yes & Yes & No & No & Yes & & \\
\hline & Follow-up ${ }^{b}$ & Yes & Yes & No & No & Yes & & \\
\hline
\end{tabular}

Note. Posttest results in bold indicate change in the desired direction. C-SA = Combined Sustained Attention; A-SA = Auditory Sustained Attention; V-SA = Visual Sustained Attention.

${ }^{a}$ Analysis of Dudley's posttest results must be interpreted with caution. ${ }^{b}$ Follow-up was conducted approximately five and a half months after posttest. ${ }^{\mathrm{C}}$ As determined by algorithms used by the IVA+Plus Interpretive Flowchart.

\section{Reading Measures}

DIBELS ORF results. Although on average reading rate improved from 85.04 words correct at baseline to 88.64 at Phase 3 , this improvement is minimal and less than expected of typical fourth graders (Figure 2). However, trend lines for accuracy indicate that all participants except Mildred exhibited some improvement in the percentage of words read correctly per minute, which means that most participants made fewer errors as the study progressed.

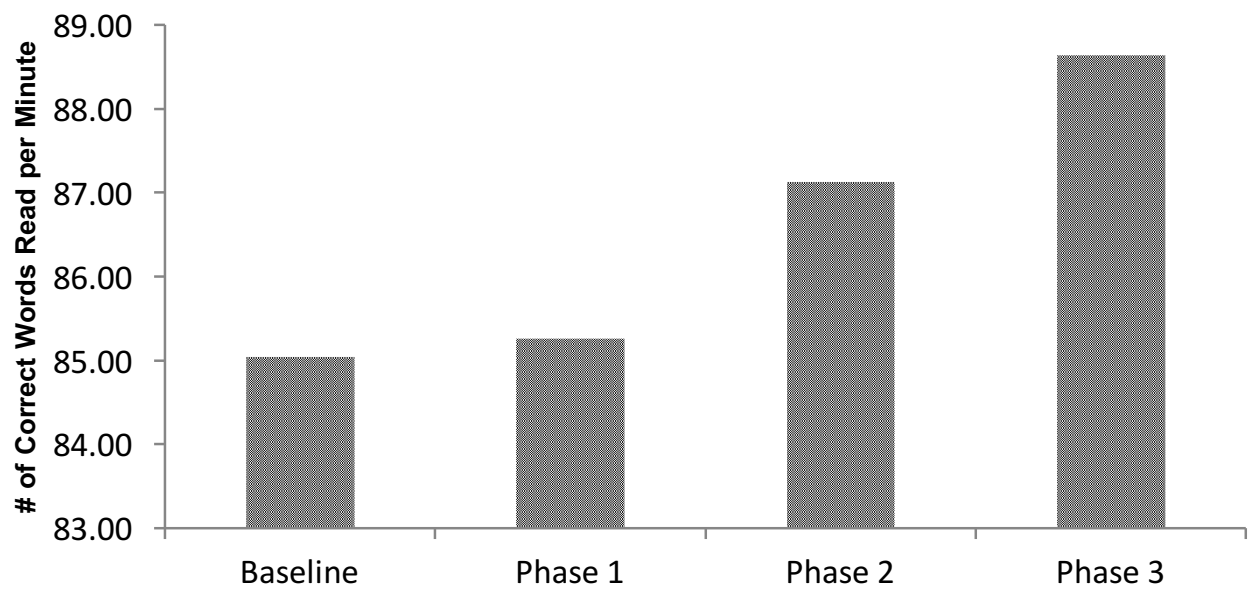

Figure 2. DIBELS ORF mean of correct words for all participants across phases. Fourth-grade students are considered "at risk" if mean words per minute $\leq 95$ at the end of the school year (University of Oregon Center on Teaching and Learning, 2012). 
AIMSweb Maze results. All participants exhibited changes in the desired direction on both AIMSweb Maze scores; the number of words correct increased and the number of errors decreased (Figure 3).
When participants' scores are combined, the mean number of correct word choices increased from 15.04 at baseline to 18.18 at Phase 3

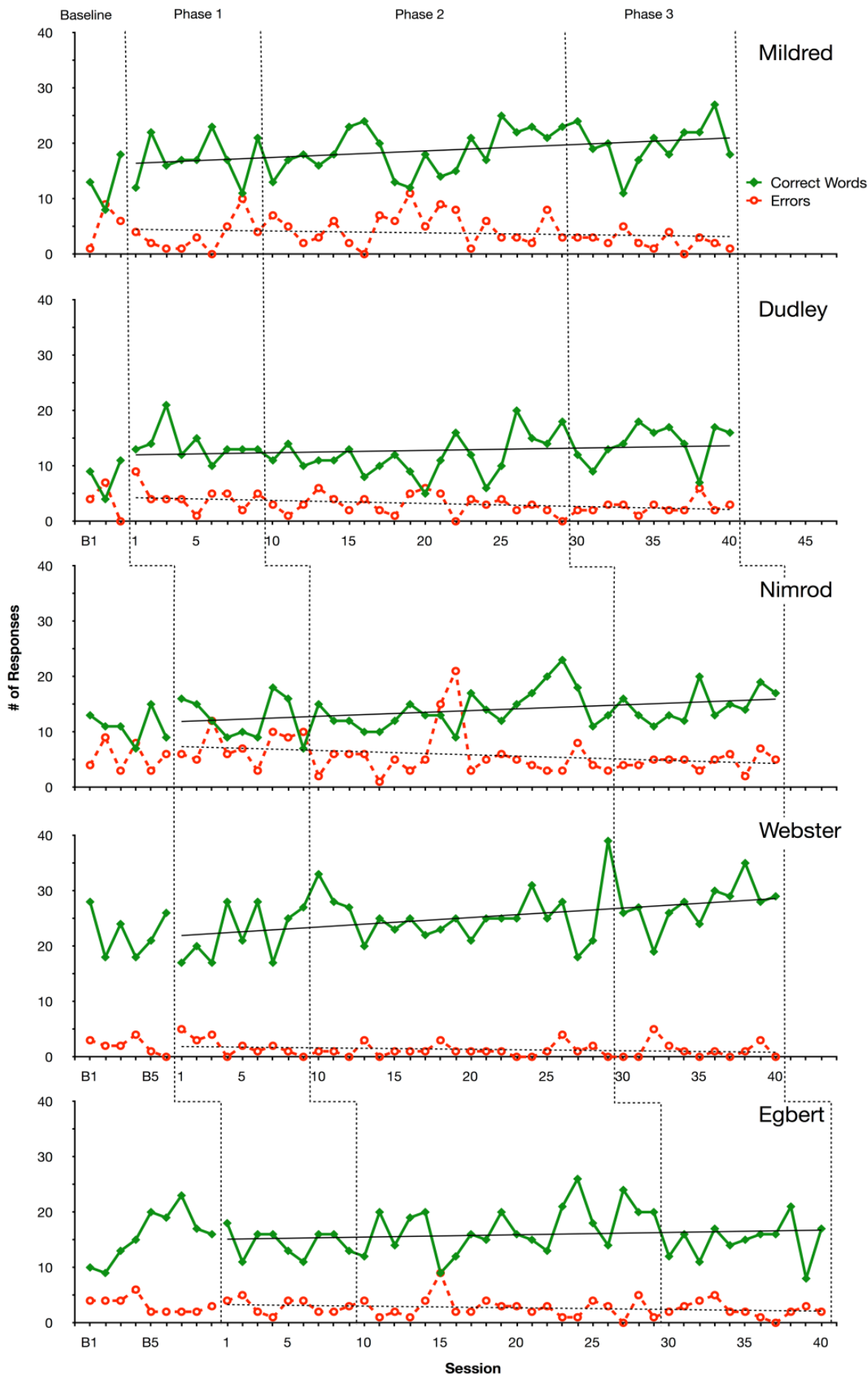

Figure 3. Maze words correct and errors, trends across all phases. Trends that were expected to increase are represented by a solid line; trends that were expected to decrease are represented by a dotted line. 
GORT-5 results. All participants except Egbert increased their ORI standard scores between pretest and posttests (Table 5). The mean standard score increased from $83(S D=9.14)$ to $90.60(S D=$ 8.32). Egbert's ORI had a slight drop from 86 to 84 ; as the standard error of measurement (SEM) on the ORI is 3 (Wiederholt \& Bryant, 2012b), this decline does not appear to be meaningful. Similar results were obtained on the fluency score, which is derived from two additional scaled scores, rate and accuracy. Four participants increased their scaled scores, while Egbert had a decrease (from 9 to 6). The mean fluency scaled score increased from 7.00 $(S D=2.12)$ to $7.60(S D=1.52)$. The mean rate score showed a slight decline, from 7.80 at pretest to 7.60 at posttest. The SEM for both the rate and fluency scores is 1 . As no participants increased or decreased \pm 1 point in their rate score at posttest, no meaningful changes occurred in rate following the intervention. The group accuracy score, however, increased from 7.00 to 8.60 , with participants gaining 1 point (Mildred), 2 points (Webster), 3 points (Nimrod), and 4 points (Dudley), except Egbert whose accuracy dropped 2 points. All participants increased their comprehension scaled scores by at least 2 points (i.e. on average from 6.80 to 9.00$)$.

\section{Table 5}

GORT-5 Pretest and Posttest Results

\begin{tabular}{|c|c|c|c|c|c|c|c|c|}
\hline & & \multicolumn{5}{|c|}{ Participant } & \multicolumn{2}{|c|}{ All Participants } \\
\hline & & Mildred & Dudley $^{a}$ & Nimrod & Webster & Egbert & Mean & $S D$ \\
\hline \multicolumn{9}{|c|}{ Oral Reading Index ${ }^{a}$} \\
\hline & Pretest & 81 & 73 & 78 & 97 & 86 & 83.00 & 9.14 \\
\hline & Posttest & 89 & 86 & 89 & 105 & 84 & 90.60 & 8.32 \\
\hline & Follow-up ${ }^{c}$ & 97 & 89 & 92 & 105 & 92 & 95.00 & 6.28 \\
\hline \multicolumn{9}{|l|}{ Rate $^{b}$} \\
\hline & Pretest & 7 & 5 & 8 & 10 & 9 & 7.80 & 1.92 \\
\hline & Posttest & 8 & 6 & 7 & 9 & 8 & 7.60 & 1.14 \\
\hline & Follow-up ${ }^{c}$ & 9 & 7 & 9 & 10 & 7 & 8.40 & 1.34 \\
\hline \multicolumn{9}{|l|}{ Accuracy $^{b}$} \\
\hline & Pretest & 6 & 4 & 6 & 9 & 10 & 7.00 & 2.45 \\
\hline & Posttest & 7 & 8 & 9 & 11 & 8 & 8.60 & 1.52 \\
\hline & Follow-up ${ }^{c}$ & 7 & 7 & 8 & 10 & 9 & 8.20 & 1.30 \\
\hline \multicolumn{9}{|l|}{ Fluency $^{\mathrm{b}}$} \\
\hline & Pretest & 6 & 4 & 7 & 9 & 9 & 7.00 & 2.12 \\
\hline & Posttest & 7 & 7 & 8 & 10 & 6 & 7.60 & 1.52 \\
\hline & Follow-up ${ }^{c}$ & 8 & 7 & 8 & 10 & 8 & 8.20 & 1.10 \\
\hline \multicolumn{9}{|l|}{ Comprehension $^{\mathrm{b}}$} \\
\hline & Pretest & 7 & 6 & 5 & 10 & 6 & $\begin{array}{l}6.80 \\
\mathbf{9 . 0 0}\end{array}$ & $\begin{array}{l}1.92 \\
173\end{array}$ \\
\hline & Posttest & 9 & 8 & 8 & $\begin{array}{l}12 \\
12\end{array}$ & $\begin{array}{l}8 \\
9\end{array}$ & $\begin{array}{c}9.00 \\
10.00\end{array}$ & $\begin{array}{l}1.73 \\
1.11\end{array}$ \\
\hline & Follow-up c & 11 & 9 & 9 & 12 & 9 & 10.00 & 1.41 \\
\hline
\end{tabular}

Note. Posttest and follow-up results in bold indicate change over previous score in the desired direction.

${ }^{a}$ Standard Scores. ${ }^{b}$ Scaled Scores (range $=1$ to 20 , mean $=10$ ). ${ }^{c}$ Follow-up was conducted approximately five and a half months after posttest.

\section{EEG/qEEG Measures}

Theta/beta results. The neurofeedback protocols used in this research were qEEG-guided and, therefore, individualized for each participant. The qEEG results for each participant reported that there were general improvements observed in each participant's EEG, with the exception of Egbert's.
Pretest and posttest qEEG theta/beta power ratios exhibited changes in the desired direction for all participants except for Dudley (Table 6). Power ratios are calculated by dividing the amplitude $(\mu \mathrm{V})$ of theta squared by the amplitude of beta squared: theta ${ }^{2} /$ beta $^{2}$. 


\section{Table 6}

qEEG Pretest and Posttest FFT Theta/Beta Power Ratios

\begin{tabular}{ccc}
\hline & \multicolumn{2}{c}{ Eyes Closed } \\
\cline { 2 - 3 } & Pretest & Posttest \\
\hline Mildred & 5.97 & $\mathbf{5 . 7 7}$ \\
Dudley & 3.88 & 4.29 \\
Nimrod & 1.97 & $\mathbf{1 . 5 2}$ \\
Webster & 4.40 & $\mathbf{3 . 6 5}$ \\
Egbert & 2.00 & $\mathbf{1 . 9 2}$ \\
\hline
\end{tabular}

Note. Posttest results in bold indicate change in the desired direction. FFT = Fast Fourier Transform. The qEEG report provided information on theta/beta power ratios calculated as (theta) $)^{2}$ ( (beta) $)^{2}$. Theta was defined as (4 to $8 \mathrm{~Hz}$ ) and beta as (13 to $21 \mathrm{~Hz}$ ).

\section{Follow-up Assessments}

Follow-up assessments (Conners 3AI, GORT-5, and the IVA+Plus) were conducted near the beginning of the following school year (November 2013), approximately five and a half months following the completion of posttest assessments. The Conners $3 \mathrm{Al}$ was again completed by parents and teachers, although teacher ratings were completed by each participant's fifth-grade teacher. Overall, teachers' ratings on the Conners 3Al-T showed improvement for four participants, with one participant (Nimrod) maintaining the score observed at posttest.

All five participants received higher scores at followup, compared to pretest results on the C-SA (Combined Sustained Attention) score (Table 4), the primary index of attention on the IVA+Plus. Two students, Nimrod and Egbert, received lower scores than obtained at posttest, although their scores remained above pretest. Similar gains were made on the subtests: all five participants received higher scores at follow-up than obtained at pretest on A-SA (Auditory Sustained Attention) with the same two students, Nimrod and Egbert, receiving lower scores than at posttest. Four of five participants made gains on V-SA (Visual Sustained Attention) with one student, Mildred, receiving a lower score than obtained at pretest. Group means for C-SA, as well as A-SA and V-SA increased from pretest to posttest, as well as from posttest to follow-up.

Positive performance was also observed on the GORT-5 at follow-up (Table 5). Four of the five participants obtained higher scores on ORI and one student maintained the score obtained at posttest.
Accuracy scores remained the same for one participant, three participants declined one scaled score although these scores remained higher than observed at pretest, and one participant (Egbert) had an increase of one scaled score although his score remained lower than at pretest. Similar to the ORI, four of the five participants' reading comprehension scores improved while one student (Webster) maintained the score he received at posttest. Because follow-up data were collected in Grade 5, GORT-5 scores are based upon the normative data for fifth-grade students, rather than fourth grade.

\section{Discussion}

A growing body of scientific literature suggests that the efficacy of neurofeedback as an intervention to assist individuals with attention deficits holds promise. Although improvements in academic performance have been observed for many decades, beginning with the seminal case study by Lubar and Shouse (1976) that noted increases in sustained attention and improvements in school performance, research has been conducted almost exclusively in clinical settings. To date, just a handful of studies have been conducted in $\mathrm{K}-12$ school settings, and all those, with the exception of Orlando and Rivera (2004), have focused exclusively on the improvement of attention with effects on academic achievement being noted only anecdotally. Thus, this study is one of the first to explicitly examine not only attention but also reading fluency and comprehension in a public school. Following 40 sessions of neurofeedback, participants demonstrated improvements on measures of attention, reading accuracy (but not fluency), and improvements on measures of reading comprehension that exceeded growth that might otherwise be attributable to maturation or regular classroom instruction.

\section{Improvements on Objective Measures of}

\section{Attention}

As predicted, our hypothesis that measurable improvements would occur in attention on objective measures (i.e., CPTs) was confirmed. In our study, the IVA+Plus was used at pretest and posttest, as well as at follow-up, as a longer measure of auditory and visual attention, and all students except Dudley (as discussed previously) showed improvement at posttest (Table 4). Furthermore, all participants, including Dudley, had higher scores at follow-up than at pretest. These findings indicate that 40 sessions of neurofeedback improved attention as predicted. 
The SAT was used following each session of neurofeedback as an objective measure to monitor changes in attention. The SAT is a hybrid of traditional CPTs (i.e., the IVA+Plus), that focus on constructs such as errors of commission and omission, as well as reaction time, and a Stroop color test, which serves as a measure of executive function. Specifically, the SAT requires participants to read a word and make a decision in response to what they have read. Thus, the assessment monitors, on a very basic level, a task associated with reading comprehension by requiring participants to correctly identify responses to written prompts. As participants made consistent gains on correctly identifying targets, with all demonstrating an increase in the number of correct responses over the course of the intervention, our results suggest that neurofeedback not only improved attention but also executive function.

\section{Effect of Neurofeedback on Reading Fluency}

The literature is essentially silent on efficacious interventions to improve fluency with ADHD populations. Theorists have long postulated that attention plays an integral role in the reading process and that it is a critical component that permits readers to derive meaning from text. The role of attention may be particularly important in assisting those whose reading abilities lack automaticity, as they must attend to the processes of reading words, rather than focus on comprehension (LaBerge \& Samuels, 1974). Furthermore, reading fluency, which combines reading rate and accuracy, is strongly correlated with reading comprehension (O'Connor et al., 2002). For these reasons, it seemed plausible to us that improved attention could influence reading rate.

That was not the case. Although neurofeedback improved attention, few changes were observed in reading fluency whether measured during progress monitoring (DIBELS ORF) or on the reading rate outcome (GORT-5, Reading Rate). Instead, all participants except Mildred exhibited improvement in the percentage of words read correctly per minute.
Thus, most participants improved their accuracy and made fewer errors as the study progressed, and it appears neurofeedback helped participants to read with more focused attention to content.

\section{Influence of Neurofeedback on Reading Comprehension}

The most encouraging outcome of this study pertains to the effect of neurofeedback on reading comprehension. While previous neurofeedback studies have reported improvements in reading comprehension incidentally to their declared dependent variables, none have explicitly examined the issue. On the progress monitoring measure (Maze) that focused on comprehension primarily at the sentence level, an examination of the means of correct word choices for all participants across phases reveals an increase in the number of correct responses, which is consistent with our finding that participants also read with increased accuracy. Specifically, the Maze results suggest that the intervention was responsible for growth beyond what would be expected. When the means of correct word choices for all participants across phases is examined, an increase is observed in the number of correct word choices identified over time (Figure 4). However, when the increases for all participants (as a group) are compared to the AIMSweb National Norms Table (NCS Pearson, 2013), which was developed with a large sample of fourth graders $(n=$ 24,881 ) and provides norms calculated at three intervals across the school year (fall, winter, and spring), participants' gains are larger than expected. Specially, the normative sample indicates that no changes are observed typically between winter and spring (e.g., the mean raw score for winter and spring are 21 correct word choices). The mean of participants' scores, between baseline ( $m=15.04$ correct word choices) and Phase 3 ( $m=18.18$ correct word choices) increased by 3.14 correct word choices. Given that the study commenced in March 2013 and concluded 12 weeks later in June 2013, suggests that neurofeedback training may have improved comprehension as measured on the Maze. 


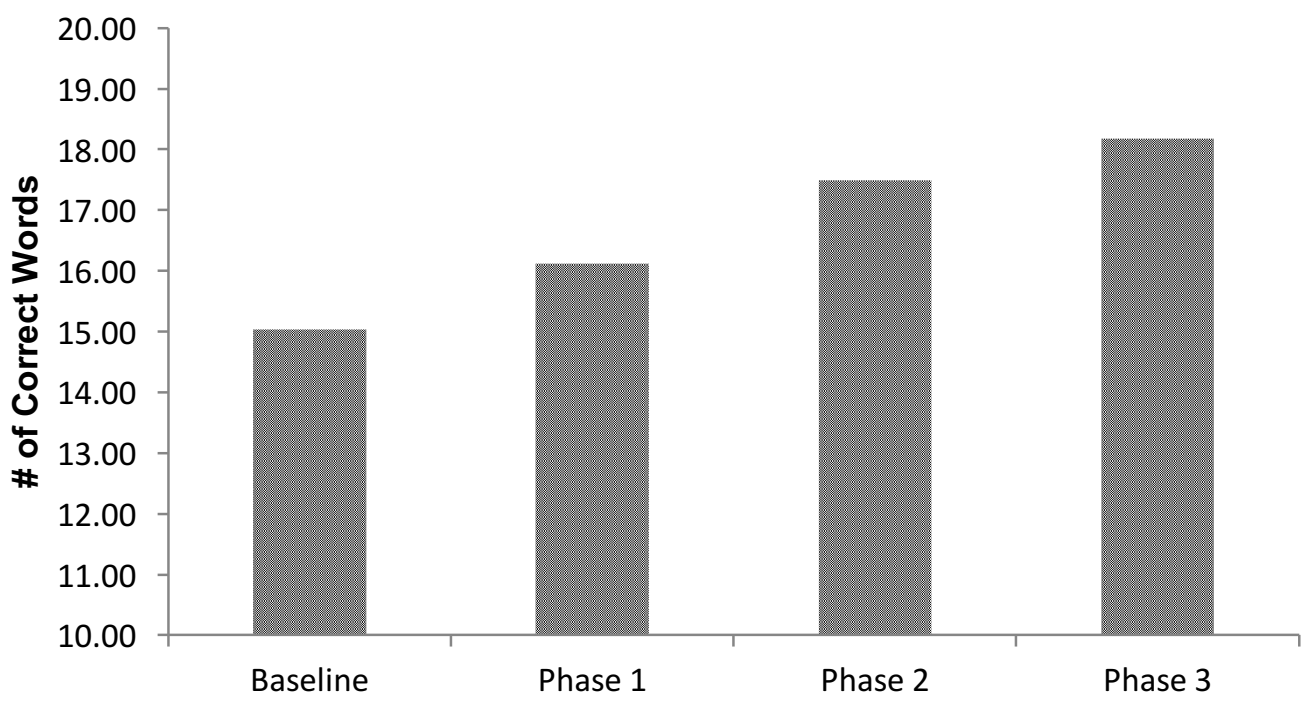

Figure 4. Maze mean of correct word choices for all participants across phases.

Perhaps more importantly, pretest and posttest of reading comprehension was measured using the GORT-5 and provided additional evidence that reading comprehension improved. After each story is read, participants answer passage-dependent questions that not only rely on the content of the text but also require them to recall what has just been read. Thus, reading as assessed on the GORT-5 provides a view commensurate with reading requirements in schools. When viewed in this context, the gains made by all students, with the exception of Egbert, indicate that reading comprehension improved following 40 sessions of neurofeedback. An evaluation of each participant's EEG also supports this conclusion; each of the five participants in this study exhibited improvements through either reduction of theta/beta ratios or normalization of EEG through improved coherence.

One issue arises in relation to measurement of changes in reading comprehension scores: The Maze assessment requires that participants read silently, while the GORT-5 requires them to read aloud. This difference presents a problem when attempting to compare the results of each assessment, because it is difficult to monitor student engagement when reading silently. Schuck (2008) observed that students with ADHD appear to read more slowly when reading silently than when reading orally and that prompting was required to keep them engaged. She concluded that participants performed significantly better on measures of comprehension while reading orally, rather than silently. In our study, during the Maze task students were observed diverting their attention elsewhere; they would look about the room or play with the pencil used for their responses. When these behaviors were evident, students were guided back to the reading task. Unfortunately, we did not include a silent reading comprehension task that had similar demands to the GORT-5; however, comparing oral and silent reading comprehension of students with ADHD could be a fruitful area for further research. The overall findings of this study suggest that neurofeedback training improves reading comprehension when given tasks that most resemble those that reflect reading for content.

\section{Limitations}

Single-case research is intended to observe the effects of an intervention to alter behavior; it seeks to establish a causal relationship between an independent variable and the dependent variables. Thus, small sample sizes are permissible and the emphasis is on the observation of effects. In keeping with SCD guidelines, this study used a sample of five students. Although effects were clearly observed, caution is advised as these results cannot be generalized to larger populations. Further research is warranted, especially since no other studies have yet directly examined the effects of neurofeedback on reading comprehension.

School schedules. Under the best of circumstances, schools are busy places and days are filled with activities. Schedules are subject to changes, some planned and others not. It is against 
this backdrop that the intensive intervention schedule of this study was overlaid. Significant events included Spring Break, as well as a week of standardized testing. Special activities included concerts, field trips, movies, plays, picnics, and other events. Although we adapted to changes in the schedule, there were times when participants' neurofeedback sessions had to be rearranged. When possible, students were scheduled as close to their normal times as possible.

\section{Social Validity}

The intensity of conducting 40 sessions of neurofeedback, particularly when training was scheduled on a daily basis, was an issue that was researched and embedded into the design of this study. The star charts and use of incentives, as described earlier, appeared to work well. As a group, participants regularly expressed satisfaction with the training sessions with several commenting that participation in the study was "awesome." Three students-Mildred, Nimrod, and Egbertasked if they were going to continue neurofeedback during the next school year. All expressed disappointment when told that the study would not continue after summer vacation.

Although the overall enthusiasm of the participants was beneficial, it was evident that at least two participants (Mildred and Egbert) also enjoyed coming to sessions because they missed class. As both of these students were generally affable and congenial, it appeared as if they especially enjoyed the individual attention received throughout the study. With both of these students, however, encouragement was regularly provided to keep them focused on doing their best during training.

\section{Implications and Future Research}

To date, only a handful of studies have examined the use of neurofeedback in public schools. This study is the one of the earliest to explicitly explore the utility of neurofeedback as an intervention to improve reading achievement in a public school setting. It is also unique in that it focused on symptoms of inattention and not hyperactivity (the samples of the other studies conducted in public schools all appear to have included children with hyperactivity/impulsivity). Specifically, this study examined the impact conditioning of EEG has on reading fluency and comprehension.

Measures of reading fluency demonstrated mixed or limited results. Other than a slight increase in accuracy, the changes in DIBELS ORF results were negligible. It is not until rate, accuracy, and fluency are examined on the longer passages found on the GORT-5 that a pattern emerges; rate remained relatively static while accuracy increased. This suggests that participants became more attentive to the text and thus read with improved accuracy (therefore, they also made fewer errors) with little or no change in rate.

The results indicate that all participants displayed increases in reading comprehension on the GORT5. Similar findings were also evident on the Maze, despite the use of considerably shorter passages as well as an assessment that does not rely on memory. Future research could examine differential performance on reading comprehension measures that rely on oral or silent reading, and on memory versus those that permit text to be reviewed, especially since all of these conditions are found in academic settings. For example, memorydependent reading comprehension skills are necessary when students read for content that must be retained, while text-dependent reading is used for assessments in the classroom and for seeking information.

Results from follow-up assessments indicate four of the five participants exhibited improvements on the primary measure of attention (C-SA) on the IVA+Plus. Furthermore, gains observed on the GORT-5 measure of reading achievement also appear to be robust. Specifically, four of the five participants achieved higher ORI and Reading Comprehension standardized scores at follow-up than observed at posttest; the remaining participant (Webster) maintained the same score on both indices as obtained at posttest. These findings imply that neurofeedback may be a viable option to assist children with attention deficits as an intervention strategy for improving both attention and reading comprehension.

While the experimental design required the use of a small sample and cannot be generalized to a larger population, this study has demonstrated potential for neurofeedback to improve educational opportunities for school children. Findings that attention improved, as measured by CPTs, are consistent with existing literature. Moreover, these improvements in attention maintained well into the next school year. Even more importantly, four of the five participants made positive gains on the GORT-5 Oral Reading Index. The one student who did not show gains on the ORI also displayed the least change in EEG; he may have been a non- or slow-responder to neurofeedback, or perhaps other issues, such as motivation, may have been involved. The overall 
findings suggest that the use of neurofeedback in a public school setting is worthy of continued exploration. The body of scientific literature on the efficacy of neurofeedback as an intervention strategy to improve the lives of individuals with attention deficits, as well as many other disorders, continues to grow. Currently, there remains a need for research in $\mathrm{K}-12$ school settings. Recent metaanalyses that indicates it is a promising intervention (Arns, de Ridder, Strehl, Breteler, \& Coenen, 2009; Hodgson, Hutchinson, \& Denson, 2012) lends support to the need for additional research. This study provides one of the early glimpses on the use of neurofeedback in a public school setting. The findings suggest that neurofeedback may be a viable option to assist children with attention deficits as an intervention strategy for improving both attention and reading achievement.

\section{Funding}

This work was supported by the Foundation for Neurofeedback and Neuromodulation Research (formerly, the International Society for Neurofeedback and Research Foundation; Student Research Grant, 2012); Brain Science International (Research Grant, 2013); and the United States Department of Education (H325D110015, 2012).

\section{References}

Akinbami, L. J., Liu, X., Pastor, P. N., \& Reuben, C. A. (2011). Attention deficit hyperactivity disorder among children aged 5-17 years in the United States, 1998-2009. NCHS Data Brief No. 70. Hyattsville, MD: National Center for Health Statistics.

American Academy of Pediatrics. (2011). ADHD: Clinical practice guideline for the diagnosis, evaluation, and treatment of attention-deficit/hyperactivity disorder in children and adolescents. Pediatrics, 128(5), 1007-1022. http://dx.doi.org /10.1542/peds.2011-2654

American Psychiatric Association. (1994). Diagnostic and statistical manual of mental disorders (4th ed.). Washington, DC: Author.

American Psychiatric Association. (2000). Diagnostic and statistical manual of mental disorders (4th ed., text rev.). Washington, DC: Author.

Arns, M., de Ridder, S., Strehl, U., Breteler, M., \& Coenen, A. (2009). Efficacy of neurofeedback treatment in ADHD: The effects on inattention, impulsivity and hyperactivity: A metaanalysis. Clinical EEG and Neuroscience, 40(3), 180-189. http://dx.doi.org/10.1177/155005940904000311

Barkley, R. A. (2002). International consensus statement on ADHD. Clinical Child and Family Psychology Review, 5(2), 89-111. http://dx.doi.org/10.1023/A:1017494719205

Barkley, R. A., Grodzinsky, G., \& DuPaul, G. J. (1992). Frontal lobe functions in attention deficit disorder with and without hyperactivity: A review and research report. Journal of Abnormal Child Psychology, 20(2), 163-188. http://dx.doi.org/10.1007/BF00916547

Bauermeister, J. J., Alegría, M., Bird, H. R., Rubio-Stipec, M., \& Canino, G. (1992). Are attentional-hyperactivity deficits unidimensional or multidimensional syndromes? Empirical findings from a community survey. Journal of the American
Academy of Child and Adolescent Psychiatry, 31(3), 423431. http://dx.doi.org /10.1097/00004583-199205000-00007

Carmody, D. P., Radvanski, D. C., Wadhwani, S., Sabo, M. J., \& Vergara, L. (2001). EEG biofeedback training and attentiondeficit/hyperactivity disorder in an elementary school setting. Journal of Neurotherapy, 4(3), 5-27. http://dx.doi.org/10.1300 IJ184v04n03_02

Chabot, R. J., \& Serfontein, G. (1996). Quantitative electroencephalographic profiles of children with attention deficit disorder. Biological Psychiatry, 40(10), 951-963. http://dx.doi.org/10.1016/0006-3223(95)00576-5

Chambless, D. L., \& Hollon, S. D. (1998). Defining empirically supported therapies. Journal of Consulting and Clinical Psychology, 66(1), 7-18. http://dx.doi.org/10.1037/0022006X.66.1.7

Chhabildas, N., Pennington, B. F., \& Willcutt, E. G. (2001). A comparison of the neuropsychological profiles of the DSM-IV subtypes of ADHD. Journal of Abnormal Child Psychology, 29(6), 529-540. http://dx.doi.org/10.1023/A:1012281226028

Coben, R., Wright, E. K., Decker, S. L., \& Morgan, T. (2015). The impact of coherence neurofeedback on reading delays in learning disabled children: A randomized controlled study. NeuroRegulation, 2(4), 168-178. http://dx.doi.org/10.15540 Inr.2.4.168

Conners, C. K. (2008a). Conners 3rd Edition. North Tonawanda, NY: Multi-Health Systems, Inc.

Conners, C. K. (2008b). Conners 3rd Edition: Manual. Toronto, ON: Multi-Health Systems, Inc.

Conners, C. K., \& Research and Development Department, MHS. (2009). Conners 3 interpretive update. Retrieved from http://downloads.mhs.com/conners/C3-Supplement.pdf

Crichton, A. (1798). On attention and its diseases. An inquiry into the nature and origin of mental derangement (Vol. 1, pp. 254290). London, England: T. Cadell, Junior, and W. Davies.

Dige, N., Maahr, E., \& Backenroth-Ohsako, G. (2008). Memory tests in subgroups of adult attention deficit hyperactivity disorder reveals simultaneous capacity deficit. International Journal of Neuroscience, 118(4), 569-591. http://dx.doi.org /10.1080/00207450701239384

Froehlich, T. E., Lanphear, B. P., Epstein, J. N., Barbaresi, W. J., Katusic, S. K., \& Kahn, R. S. (2007). Prevalence, recognition, and treatment of attention-deficit/hyperactivity disorder in a national sample of US children. Archives of Pediatrics and Adolescent Medicine, 161(9), 857-864. http://dx.doi.org /10.1001/archpedi.161.9.857

Ghelani, K., Sidhu, R., Jain, U., \& Tannock, R. (2004). Reading comprehension and reading related abilities in adolescents with reading disabilities and attention-deficit/hyperactivity disorder. Dyslexia, 10(4), 364-384. http://dx.doi.org/10.1002 /dys. 285

Goldman, L. S., Genel, M., Bezman, R. J., \& Slanetz, P. J. (1998). Diagnosis and treatment of attention-deficit/hyperactivity disorder in children and adolescents. Journal of the American Medical Association, 279(14), 1100. http://dx.doi.org/10.1001 /jama.279.14.1100

Good, R. H., \& Kaminski, R. A. (2003). Dynamic Indicators of Basic Early Literacy Skills (DIBELS) 6th Edition (6th ed.). Longmont, CO: Sopris West Educational Services.

Gruzelier, J., \& Egner, T. (2005). Critical validation studies of neurofeedback. Child and Adolescent Psychiatric Clinics of North America, 14(1), 83-104. http://dx.doi.org/10.1016 j.chc.2004.07.002

Gualtieri, C. T., \& Johnson, L. G. (2006). Reliability and validity of a computerized neurocognitive test battery, CNS Vital Signs. Archives of Clinical Neuropsychology, 21(7), 623-643. http://dx.doi.org/10.1016/j.acn.2006.05.007

Gustafson, S. A., Nassar, S. L., \& Waddell, D. E. (2011). Singlecase design in psychophysiological research. Part I: Context, structure, and techniques. Journal of Neurotherapy, 15(1), 18-34. http://dx.doi.org/10.1080/10874208.2011.545762 
Hall, A. H., \& Tannebaum, R. P. (2013). Test Review: Gray Oral Reading Tests-Fifth Edition. Journal of Psychoeducational Assessment, 31(5), 516-520. http://dx.doi.org/10.1177 10734282912468578

Hart, E. L., Lahey, B. B., Loeber, R., Applegate, B., \& Frick, P. J. (1995). Developmental change in attention-deficit hyperactivity disorder in boys: a four-year longitudinal study. Journal of Abnormal Child Psychology, 23(6), 729-749. http://dx.doi.org/10.1007/BF01447474

Hodgson, K., Hutchinson, A. D., \& Denson, L. (2012). Nonpharmacological treatments for ADHD: A meta-analytic review. Journal of Attention Disorders, 20(10), 1-8. http://dx.doi.org/10.1177/1087054712444732

Horner, R. H., Carr, E. G., Halle, J., McGee, G., Odom, S., \& Wolery, M. (2005). The use of single-subject research to identify evidence-based practice in special education. Exceptional Children, 71(2), 165-179. http://dx.doi.org $/ 10.1177 / 001440290507100203$

Jasper, H. H. (1958). The ten-twenty electrode system of the International Federation. Electroencephalography and Clinical Neurophysiology, 10(2), 371-375.

Keenan, J. M., \& Betjemann, R. S. (2006). Comprehending the Gray Oral Reading Test without reading it: Why comprehension tests should not include passageindependent items. Scientific Studies of Reading, 10(4), 363380.

Kennedy, C. H. (2005). Single-case designs for educational research. Boston, MA: Allyn and Bacon.

Kratochwill, T. R., Hitchcock, J., Horner, R. H., Levin, J. R., Odom, S. L., Rindskopf, D. M., \& Shadish, W. R. (2010). Single-case designs technical documentation. What Works Clearinghouse. Retrieved from http://ies.ed.gov/ncee/wwc/pdf /reference_resources/wwc_scd.pdf

LaBerge, D., \& Samuels, S. J. (1974). Toward a theory of automatic information processing in reading. Cognitive Psychology, 6(2), 293-323. http://dx.doi.org/10.1016/00100285(74)90015-2

Leins, U., Goth, G., Hinterberger, T., Klinger, C., Rumpf, N., \& Strehl, U. (2007). Neurofeedback for children with ADHD: A comparison of SCP and theta/beta protocols. Applied Psychophysiology and Biofeedback, 32(2), 73-88. http://dx.doi.org/10.1007/s10484-007-9031-0

Linden, M., Habib, T., \& Radojevic, V. (1996). A controlled study of the effects of EEG biofeedback on cognition and behavior of children with attention deficit disorder and learning disabilities. Biofeedback and Self-regulation, 21(1), 35-49. http://dx.doi.org/10.1007/BF02214148

Lubar, J. F. (1991). Discourse on the development of EEG diagnostics and biofeedback for attention-deficit/hyperactivity disorders. Biofeedback and Self-regulation, 16(3), 201-225. http://dx.doi.org/10.1007/BF01000016

Lubar, J. F., \& Shouse, M. N. (1976). EEG and behavioral changes in a hyperkinetic child concurrent with training of the sensorimotor rhythm (SMR). Biofeedback and Self-regulation, 1(3), 293-306. http://dx.doi.org/10.1007/bf01001170

Mitsar EEG-201, WinEEG [Apparatus and software]. Saint Petersburg, Russia. Mitsar Co. Ltd. Retrieved from http://www.mitsar-medical.com/eeg-software/qeeg-software/

Monastra, V. J., Lynn, S., Linden, M., Lubar, J. F., Gruzelier, J., \& LaVaque, T. J. (2005). Electroencephalographic biofeedback in the treatment of attention-deficit/hyperactivity disorder. Applied Psychophysiology and Biofeedback, 30(2), 95-114. http://dx.doi.org/10.1007/s10484-005-4305-x

Monastra, V. J., Monastra, D. M., \& George, S. (2002). The effects of stimulant therapy, EEG biofeedback, and parenting style on the primary symptoms of attentiondeficit/hyperactivity disorder. Applied Psychophysiology and Biofeedback, 27(4), 231-249. http://dx.doi.org/10.1023 IA:1021018700609
National Center on Response to Intervention. (2012). Curriculum based measurement in reading: Maze fluency. Retrieved from http://www.rti4success.org/progressMonitoringTools. Accessed May 20, 2012.

NCS Pearson. (2013). AIMSweb national norms table: MAZE comprehension. Retrieved from https://aimsweb.pearson.com/

Nigg, J. T. (2005). Neuropsychologic theory and findings in attention-deficit/hyperactivity disorder: The state of the field and salient challenges for the coming decade. Biological Psychiatry, 57(11), 1424-1435. http://dx.doi.org/10.1016 /j.biopsych.2004.11.011

O'Connor, R. E., Bell, K. M., Harty, K. R., Larkin, L. K., Sackor, S. M., \& Zigmond, N. (2002). Teaching reading to poor readers in the intermediate grades: A comparison of text difficulty. Journal of Educational Psychology, 94(3), 474-485. http://dx.doi.org/10.1037//0022-0663.94.3.474

Orlando, P. C., \& Rivera, R. O. (2004). Neurofeedback for elementary students with identified learning problems. Journal of Neurotherapy, 8(2), 5-19. http://dx.doi.org/10.1300 /j184v08n02_02

Rossiter, T. (2002). Neurofeedback for AD/HD: A ratio feedback case study and tutorial. Journal of Neurotherapy, 6(3), 9-35. http://dx.doi.org/10.1300/j184v06n03_03

Sandford, J. A. (2012). SmartMind Pro. Richmond, VA: BrainTrain, Inc.

Sandford, J. A., \& Turner, A. (2007). Integrated Visual and Auditory Continuous Performance Test (IVA+Plus). Richmond, VA: BrainTrain, Inc.

Sandford, J. A., \& Turner, A. (2009). Integrated Visual and Auditory Continuous Test: Interpretation manual. Richmond, VA: BrainTrain, Inc.

Schuck, S. E. B. (2008). Reading comprehension and the role of oral and silent reading: Comparing children with and without attention deficit hyperactivity disorder (Doctoral dissertation). University of California, Riverside.

Shanahan, T. (2005). [Review of the DIBELS: Dynamic Indicators of Basic Early Literacy Skills, Sixth Edition]. In R. A. Spies \& B. S. Plake (Eds.), The sixteenth mental measurements yearbook (pp. 310-312). Lincoln, NE: Buros Institute of Mental Measurements.

Shinn, M. R., \& Shinn, M. M. (2002a). AIMSweb training workbook: Administration and scoring of Reading CurriculumBased Measurement (R-CBM). Eden Prairie, MN: Edformation, Inc.

Shinn, M. R., \& Shinn, M. M. (2002b). AIMSweb training workbook: Administration and scoring of reading maze for use in general outcome measurement. Eden Prairie, MN: Edformation, Inc.

Snyder, S. M., \& Hall, J. R. (2006). A meta-analysis of quantitative EEG power associated with attention-deficit hyperactivity disorder. Journal of Clinical Neurophysiology, 23(5), 441-456. http://dx.doi.org/10.1097/01.wnp.0000221363.12503.78

Steiner, N. J., Frenette, E. C., Rene, K. M., Brennan, R. T., \& Perrin, E. C. (2014). Neurofeedback and cognitive attention training for children with attention-deficit hyperactivity disorder in schools. Journal of Developmental and Behavioral Pediatrics, 35(1), 18-27. http://dx.doi.org/10.1097 /DBP.0000000000000009

Still, G. F. (1902a). Some abnormal psychical conditions in children: Lecture 1. The Lancet, 159(4102), 1008-1012. http://dx.doi.org/10.1016/s0140-6736(01)74984-7

Still, G. F. (1902b). Some abnormal psychical conditions in children: Lecture 2. The Lancet, 159(4103), 1077-1082. http://dx.doi.org/10.1016/S0140-6736(01)70022-0

Still, G. F. (1902c). Some abnormal psychical conditions in children: Lecture 3. The Lancet, 159(4104), 1163-1168. http://dx.doi.org/10.1016/S0140-6736(01)74901-X

Swanson, J. M., Posner, M., Potkin, S. G., Bonforte, S., Youpa, D. Fiore, C., ... Crinella, F. (1991). Activating tasks for the study 
of visual-spatial attention in ADHD children: A cognitive anatomic approach. Journal of Child Neurology, 6(1), S119S127. http://dx.doi.org/10.1177/0883073891006001121

U.S. Department of Education, Office of Special Education and Rehabilitative Services, \& Office of Special Education Programs. (2008). Identifying and treating attention deficit hyperactivity disorder: A resource for school and home. Washington, DC: Author.

University of Oregon Center on Teaching and Learning. (2012). DIBELS benchmark goals: Three assessment periods per year. Retrieved from https://dibels.uoregon.edu/docs /benchmarkgoals.pdf

Vernon, D., Egner, T., Cooper, N., Compton, T., Neilands, C., Sheri, A., \& Gruzelier, J. (2003). The effect of training distinct neurofeedback protocols on aspects of cognitive performance. International Journal of Psychophysiology, 47(1), $\quad$ 75-85. http://dx.doi.org/10.1016/S01678760(02)00091-0

Volkow, N. D., Wang, G.-J., Newcorn, J. H., Kollins, S. H., Wigal, T. L., Telang, F., ... Swanson, J. M. (2011). Motivation deficit in ADHD is associated with dysfunction of the dopamine reward pathway. Molecular Psychiatry, 16(11), 1147-1154. http://dx.doi.org/10.1038/mp.2010.97

Wadhwani, S., Radvanski, D. C., \& Carmody, D. P. (1998) Neurofeedback training in a case of attention deficit hyperactivity disorder. Journal of Neurotherapy, 3(1), 42-49. http://dx.doi.org/10.1300/J184v03n01 05

Wechsler, D. (2011). Wechsler Abbreviated Scale of IntelligenceSecond Edition Manual. Bloomington, MN: Pearson Education, Inc.
Weiler, M. D., Bernstein, J. H., Bellinger, D. C., \& Waber, D. P. (2000). Processing speed in children with attention deficit/hyperactivity disorder, inattentive type. Child Neuropsychology, 6(3), 218-234. http://dx.doi.org/10.1076 /chin.6.3.218.3156

Wiederholt, J. L., \& Bryant, B. R. (2001). Gray Oral Reading TestFourth Edition (GORT-4). Austin, TX: Pro-Ed.

Wiederholt, J. L., \& Bryant, B. R. (2012a). Gray Oral Reading Test-Fifth Edition (GORT-5). Austin, TX: Pro-Ed.

Wiederholt, J. L., \& Bryant, B. R. (2012b). Gray Oral Reading Test-Fifth Edition (GORT-5): Examiner's manual (5th ed.). Austin, TX: Pro-Ed.

Willcutt, E. G., \& Pennington, B. F. (2000). Comorbidity of reading disability and attention-deficit/hyperactivity disorder: Differences by gender and subtype. Journal of Learning Disabilities, 33(2), 179-191. http://dx.doi.org/10.1177 /002221940003300206

Woodcock, R. (2011). Woodcock Reading Mastery Tests, Third Edition. San Antonio, TX: Pearson Education, Inc.

Woodcock, R. W., McGrew, K. S., \& Mather, N. (2007). Woodcock-Johnson III Tests of Achievement. Rolling Meadows, IL: Riverside Publishing.

Received: February 5, 2016

Accepted: May 29, 2016

Published: June 21, 2016 